\title{
Study on detection and identification of some Mycobacterium species by using some bacteriological and immunological techniques
}

\author{
Mohamed I. Abou-Dobara*, Amira A. El-Fallal, Nouran S. Almelegy \\ Botany and Microbiology Department, Faculty of Sciences, Damietta University, Egypt
}

Received: 11 May 2016/ Accepted: 2 August 2016

*Corresponding author: aboudobara@gmail.com

\begin{abstract}
This study aimed to compare and analyze the results of microbiological and immunological laboratory investigations of pulmonary tuberculosis and non-tuberculosis patients from Hospitals at Saudi Arabia, and also to evaluate the serodiagnosis detection of tuberculosis promptly using immunological assays in comparison with the traditional Ziehl Neelsen microbiological techniques. This is to develop reliable, effective assay, shorter duration and more specific diagnosis of active tuberculosis. The sensitivity, specificity and efficiency of Ziehl-Neelsen stain were $48 \%, 100 \%$ and $64 \%$, respectively. An identified antigen in serum samples of infected persons with pulmonary tuberculosis was detected using TB-Spot ELISA technique. The sensitivity, specificity and efficiency of TB-Spot ELISA for the detection of antigen were $97 \%, 95 \%$ and $98 \%$, respectively. The serum levels of TNF- $\alpha$ reach statistical difference $(\mathrm{P}<0.0001)$ in patients positive for TB antigen in comparison with the levels in negative individuals for TB.

Keywords: pulmonary tuberculosis, QuantiFERON-TB Gold In-Tube, ELISA, interferon gamma release assays (IGRAs).
\end{abstract}

\section{Introduction}

Tuberculosis is caused by Mycobacterium tuberculosis (MTB) as well as Nontubercular Mycobacterium (NTM) with similar clinical presentation. Infections due to NTM were reported to have increased in the past few years ${ }^{[1]}$.

In recent decades, the burden of tuberculosis (TB) has been increasingly falling on developing countries. Although the TB vaccine Bacille Calmette-Guérin (BCG) is broadly vaccinated and DOTS (Directly Observed Treatment, Shortcourse) program is well implemented, the incidence rate of active tuberculosis (ATB) in China has been doubled over ten years (39.03/100,000 in 1999 vs. 81.09/100,000 in 2009), with the death rate soaring 7-fold in this decade ${ }^{[2]}$.

The last decade has witnessed significant advances in mycobacterial genomics and cellular research which have 
resulted in the development of two new blood tests, the enzyme-linked immunospot assay (ELISpot) (TSPOT.TB, Oxford Immunotec, Oxford, UK) and the enzymelinked immunosorbent assay (ELISA) (QuantiFERON-TB Gold In-Tube, Cellestis, Carnegie, Australia. ${ }^{[3]}$ These tests, which are collectively known as interferon gamma release assays (IGRAs), detect latent tuberculosis infection (LTBI) by measuring interferon (IFN)-gamma release in response to antigens present in Mycobacterium tuberculosis, but not bacille Calmette-Guerin (BCG) vaccine and most nontuberculous mycobacteria. This is done through enumeration of IFNgamma-secreting $\mathrm{T}$ cells (ELISpot) or by measurement of IFN-gamma concentration (ELISA). The evidence base for these tests has expanded rapidly and now demonstrates that IGRAs are more specific than the tuberculin skin test (TST) as they are not confounded by previous BCG vaccination. In addition, with active tuberculosis (TB) as a surrogate for LTBI, it appears that the ELISA has a similar sensitivity to the TST, whereas the ELISA pot is more sensitive Using the degree of exposure to TB as a surrogate for LTBI, both assays correlate at least as well with TB exposure as the TST ${ }^{[3]}$. The aim of this work is to detect and identify some Mycobacterium species from some clinical samples by using some bacteriological and immunological techniques.

\section{Materials and methods:}

Sputum and serum samples of 322 individuals (234 males, 88 females; aged 25-55 year) were collected from Hospitals at Saudi Arabia. Sputum smear were examined using Ziehl Neelsen stain for detection of acid-fast bacilli. Blood samples were allowed to clot for separation of sera. Tubes were centrifuged at 4000 rpm for 10 minutes, and the sera were separated and stored at $-20{ }^{\circ} \mathrm{C}$. Patients with pulmonary $M$. tuberculosis TB ( $\mathrm{n}=$ 207) were diagnosed by sputum smear for acid-fast bacilli, radiographic evidence or clinical symptoms. In addition, sera of patients admitted to the hospital for a defined acute or chronic non-tuberculous diseases $\quad(n=93)$ including; chronic obstructive pulmonary disease $(\mathrm{n}=34)$, asthma ( $\mathrm{n}=15)$, ischemic heart disease $(n=7)$, pneumonia $(n=5)$, bronchitis $(n=3)$, lung cancer $(\mathrm{n}=2)$ and lung infection $(\mathrm{n}=5)$ as well as sera of healthy volunteers $(\mathrm{n}=$ 22) with no signs of clinical impairment and normal chest radiographs were included as controls.

\section{Method of QFT-GIT ${ }^{[4]}$}

Blood from each subject was collected at one morning visit into two sets of QFT-GIT tubes (Set 1 or Set 2) so that an automated ELISA and a manual ELISA could be performed from each set of tubes. Tubes were purchased from Cellestis, Ltd (Cellestis Limited, Carnegie, Victoria, Australia), and each set of tubes included a Nil tube, a TB antigen tube, and a Mitogen tube. Each tube was labeled with a number and a barcode that:

(1) Identified the specimen.

(2) Identified the tube type (i.e., Nil tube, TB antigen tube, or Mitogen tube).

(3) Linked the specimen to subject and collection information.

One $\mathrm{mL}$ of blood was collected into each tube and tube contents were mixed with a Stuart rock and roll mixer (SciTech Instruments, Inc., Franklin, $\mathrm{NJ}$ ) for 3 minutes at $33 \mathrm{RPM}$. Within one hour of blood collection, tubes were incubated at $37 \pm 0.5^{\circ} \mathrm{C}$ for 23 to 24 hours and then centrifuged at 3,000 g for 10 minutes.

IFN- $\gamma$ concentrations in plasmas in Nil tubes (Nil), TB antigen tubes (TB), and Mitogen tubes (Mitogen) were determined by ELISAs performed on the day after blood collection using reagents included in QFT-GIT kits. ELISAs were performed with the aid of an automated ELISA workstation (automated ELISA) or without the aid of an automated ELISA workstation (manual ELISA).

For manual ELISAs, reagents were dispensed with single and multichannel pipetters (Rainin Instrument, LLC, Oakland, CA); plates were washed with a Biotrak II Microplate washer (Biochrom, Ltd., Cambridge, UK), and optical densities (ODs) were measured with a Thermo Scientific, Multiskan Ascent (Waltham, MA) 
IFN- $\gamma$ standards from QFT-GIT kits were serially diluted and eight IFN- $\gamma$ concentrations (i.e., 8, 4, 2, 1, 0.5, 0.25, 0.125 , and $0 \mathrm{IU} / \mathrm{mL}$ ) were used in duplicate to create a standard curve for each ELISA. OD values were imported electronically, and plasma IFN- $\gamma$ concentrations were determined using a Microsoft Access database (Microsoft, Inc., Seattle, WA) developed at CDC. ELISAs not meeting quality specifications as defined by the manufacturer were immediately repeated. TB Responses were calculated by subtracting Nil from $\mathrm{TB}$, and Mitogen Responses were calculated by subtracting Nil from Mitogen.

\section{METHOD of TB SPOT}

Step 1 -Principle of assay and Rationale for a T cell-based approach ${ }^{[5]}$

M. tuberculosis: The intracellular pathogen is difficult to recover from infected subjects. Antibody response in M. tuberculosis infection is weak. MTB infection evokes a strong Th1 cellmediated immune response. MTB-specific $\mathrm{T}$ cells might be an accurate marker of infection. T-cells from individuals infected with MTB become sensitized to specific antigens (ESAT-6 and CFP10) in vivo, when T-cells re-encounter these antigens $e x$ vivo they become stimulated and produce cytokines - INF-Gamma. After incubation and detection such T-cells produce dark spots, 1 Dark Spot $=1$ individual $M$. tuberculosis specific T-cell. The readout is simply the number of spots. Simplified ELISPOT Overnight blood test that detects both latent TB infection (LTBI) and active TB disease, detects IFN $\gamma$ released by $\mathrm{T}$ cells stimulated with specific TB antigens.

\section{T-SPOT ${ }^{\circledR} . T B($ Oxford Immunotec UK)}

Ninety six wells plate, 2 antigens MTBspecific (ESAT-6 and CFP10) in 2 separate wells, single cut-off at 6 SFC (any antigen) Sample Collection and Preparation: Vacutainer Cell Preparation Tubes were used.

Blodd was collected in $4 \mathrm{~mL}$ tubes was centrifuged at $18-25^{\circ} \mathrm{C} 1800 \times \mathrm{xg}$ for 30 minutes
Ficoll Extraction: blood, that was collected into Lithium Heparin tubes, was diluted 1:1 with RPMI 1640 (pre-warmed to $37^{\circ} \mathrm{C}$ ). -Carefully layer sample was put onto Ficoll-Paque $^{\mathrm{TM}}$ Plus (Approx ratio - 3:1) and not allowed to mix. Then was centrifuged at $1000 \mathrm{xg}$ for 22 minutes at room temperature $\left(18-25^{\circ} \mathrm{C}\right)$ and the white cloudy band of PBMCs was removed from the CPTs or Ficoll extraction tube and was transferred into sterile $15 \mathrm{~mL}$ conical centrifuge tube

\section{Step 2 - Cell washing and counting}

Cell washing with culture media was performed to remove endogenous cytokine and removes background INF-Gamma.

- $\quad$ RPMI 1640 or AIM-V ${ }^{\circledR}$ was used for PBMC wash steps (warmed to $37^{\circ} \mathrm{C}$ )

- Different culture media can be used in this step; these are:

1. AIM-V is highly nutritious medium, maintains healthy, functional cells

2. RPMI 1640 is less nutritious which is Less expensive than AIM-V and NOT suitable for the overnight incubation of T-cells

\section{Step 3 - Plate Set up and Incubation}

- Four wells were required for each patient sample and the samples were vertically arranged on the plate as below:

- $\quad 50 \mu \mathrm{L}$ AIM V to each Nil Control well

- $50 \mu \mathrm{L}$ Panel A (ESAT-6) to each relevant well

- $50 \mu \mathrm{L}$ Panel B (CFP10) to each relevant well

- $50 \mu \mathrm{L}$ Positive Control (PHA) to each Positive Control well

To each 4 wells used per patient sample, $100 \mu \mathrm{L}(250,000$ viable cells $)$ of cell dilution was added and incubated at $37^{\circ} \mathrm{C}$ (humidified \& $5 \% \mathrm{CO}_{2}$ ) for $16-20$ hours

\section{Step 4 - Wash and add secondary antibody}

Spot Development and Counting The plate was removed from the incubator, then well contents was discarded, and was washed 4 times with $200 \mathrm{~mL}$ freshly prepared or sterile $1 \mathrm{x}$ PBS. $50 \mu \mathrm{L}$ working strength Conjugate Reagent was added to all wells. The plate was 
incubated for 1 hour at $2-8^{\circ} \mathrm{C}$. Wells were washed 4 times with $200 \mathrm{~mL} 1 \mathrm{x}$ PBS to remove any unbound Conjugate Reagent. The $50 \mu \mathrm{L}$ substrate solution was added to each well. The wells were incubated at room temperature for 7 minutes, then spots were examined. The plate was washed thoroughly with distilled or deionised water to stop detection reaction. Plates must be thoroughly dried before counting spots. The plate was allowed to dry in the dark, either by placing the plate:

- In a dry oven at up to $37^{\circ} \mathrm{C}$ for four hours

- Or In the air flow of a laminar flow hood

- Or in a well-ventilated area at room temperature overnight

\section{Counting spots}

Spots were counted using a magnifying glass, dissecting microscope. Alternatively an automated ELISPOT imager was used. T-SPOT. TB requires 250,000 (+/- 50,000) viable PBMCs per well, 4 wells are required for each patient sample. 1,000,000 cells required per test sample.

\section{Results}

Sputum samples of 322 individuals were investigated in the present study. They were obtained from patients with pulmonary TB $(n=207)$, as well as patients with respiratory diseases rather than $\mathrm{TB}(\mathrm{n}=$ 93) and healthy controls $(n=22)$. Sputum samples from 207 clinically diagnosed as tuberculosis patients were screened for the detection of M. tuberculosis using ZiehlNeelsen stain.

Sensitivity of tuberculin skin test (TST) against clinical examination as gold standard method.

We take only 50 patients to do the test and there were positive for TST test 43 cases $(86 \%)$ and 7 cases $(14 \%)$ were smeared negative, which indicates the sensitivity of TST test was $86 \%$ using clinical examination as the gold standard method (Table 1).
Table 1. Sensitivity of TST test against clinical examination as gold standard method of 207 cases with pulmonary TB.

\begin{tabular}{|ll|l|l|}
\hline +ve & TST & -ve TST & Sensitivity \\
\hline 43 & cases $\quad(86$ & 7 cases $(14 \%)$ & $75 \%$ \\
$\%)$ & & & \\
\hline
\end{tabular}

Specificity of tuberculin skin test (TST) against clinical examination as gold standard method.

All cases of clinically diagnosed as respiratory diseases other than TB $(n=50)$ as well as healthy controls $(n=22)$ were smeared negative for M. tuberculosis. 30 cases were examined and the specificity of tuberculin skin test (TST) assumed to be $100 \%$.

Sensitivity of Ziehl-Neelsen stain against clinical examination as the gold standard method.

Of the 207 cases with pulmonary tuberculosis, 99 sputum smear were positive for M. tuberculosis (48\%) and 108 cases $(52 \%)$ were smeared negative, which indicates the sensitivity of Ziehl-Neelsen stain was $48 \%$.

\section{Specificity of Ziehl-Neelsen stain}

All sputum samples from clinically diagnosed as respiratory diseases other than TB $(n=93)$ as well as healthy controls $(n=$ 22) were smeared negative for $M$. tuberculosis. So the specificity of ZiehlNeelsen stain assumed to be $100 \%$.

\section{Efficiency of Ziehl-Neelsen stain}

The 207 cases with pulmonary tuberculosis, 99 sputum smear were true positive for $M$. tuberculosis (48\%). All sputum samples from 93 clinically diagnosed as controls were true negative for $M$. tuberculosis. So the efficiency of Ziehl-Neelsen stain was $64 \%$ against clinical examination as the gold standard method.

\section{TB SPOT RESULT}

- T-SPOT. TB requires 250,000 (+/$50,000)$ viable PBMCs per well 
- 4 wells are required for each patient sample

- $\quad 1,000,000$ cells required per test sample

REACTIVE: Indicates that the sample contains T cells reactive to $M$. tuberculosis, figure 1.

NOT REACTIVE: Indicates that the sample probably does not contain $\mathrm{T}$ cells reactive to $M$. tuberculosis, figure 1 .

INDETERMINATE: The test was repeated to confirm the result

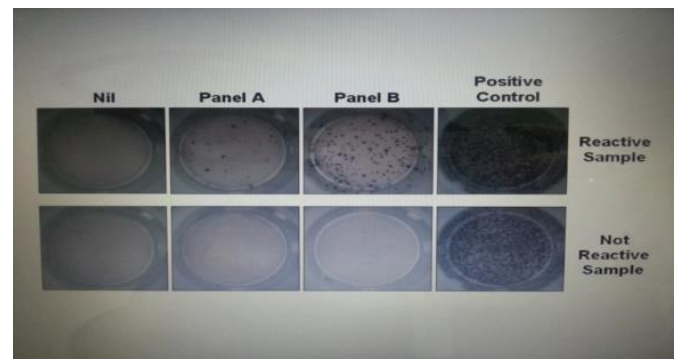

Figure 1; Positive \& negative reaction of tbspot.

Serum samples of patients with pulmonary TB $(n=98)$ were tested for TB-SPOT. Of 98 pulmonary tuberculosis cases, 95 (97\%) were positive for tuberculosis antigen and 3 (3\%) cases were negative for tuberculosis antigen.(Table 2) So the sensitivity of TBSPOT for the detection of TB antigen 97\% using clinical examination as the gold standard method.

Table 2. Sensitivity of of TB-SPOT for the detection of TB antigen in 98 cases with pulmonary $\mathrm{TB}$.

\begin{tabular}{|c|c|c|}
\hline +ve TB antigen & -ve TB antigen & Sensitivity \\
\hline $95(97 \%)$ & $3(3 \%)$ & $97 \%$ \\
\hline
\end{tabular}

The tuberculosis antigen was detected in 3 out of 60 serum samples of controls (5\% false positive). So the specificity of $\mathrm{TB}$ SPOT for the detection of TB antigen was $95 \%$ using clinical examination as the gold standard method (Table 3). The efficiency of TB-Spot for the detection of TB antigen was $96 \%$ (Table 4).

Table 3. Specificity of TB-SPOT for the detection of TB antigen in 60 case controls

\begin{tabular}{|c|c|c|}
\hline +ve TB antigen & -ve TB antigen & Specificity \\
\hline $3(5 \%)$ & $57(95 \%)$ & $95 \%$ \\
\hline
\end{tabular}

Table 4. Efficiency of TB SPOT for the detection of TB antigen in 98 cases with pulmonary TB and 60 cases controls

\begin{tabular}{|c|c|c|}
\hline Sensitivity & Specificity & Efficiency \\
\hline $\begin{array}{c}\text { 95 of 98cases } \\
\text { pulmonary TB } \\
\text { were + ve TB } \\
\text { antigen }\end{array}$ & $\begin{array}{c}57 \text { of } 60 \text { cases } \\
\text { Controls were- } \\
\text { ve TB antigen } \\
(97 \%)\end{array}$ & $96 \%$ \\
\hline
\end{tabular}

Serum samples of patients with pulmonary TB were tested for (QFT-G) test Of 60 pulmonary tuberculosis cases 57 (95\%) were positive for tuberculosis antigen and $3(5 \%)$ cases were negative for tuberculosis antigen. So the sensitivity of (QFT-G) test for the detection of TB antigen (95\%).The tuberculosis antigen was detected in 1 out of 30 serum samples of controls. So the specificity of (QFT-G) test for the detection of TB antigen was 97 $\%$ (Table 5).

Table 5. Specificity of (QFT-G) test for the detection of TB antigen in 30 case controls

\begin{tabular}{|c|c|c|}
\hline $\begin{array}{c}\text { +ve TB } \\
\text { antigen }\end{array}$ & $\begin{array}{c}\text {-ve TB } \\
\text { antigen }\end{array}$ & Specificity \\
\hline $1(3 \%)$ & $29(97 \%)$ & $97 \%$ \\
\hline
\end{tabular}

The efficiency of (QFT-G) test for the detection of $T B$ antigen:

Efficiency can be expressed through sensitivity and specificity (Table 6).

Table 6. Efficiency of (QFT-G) test for the detection of $\mathrm{TB}$ antigen in 50 cases with pulmonary TB and 30 cases controls.

\begin{tabular}{|c|c|c|}
\hline Sensitivity & Specificity & Efficiency \\
\hline 57 of 60 cases & 29 of 30 cases & $95.5 \%$ \\
pulmonary TB & Controls were- & \\
were + ve TB & ve TB antigen & \\
antigen & $(97 \%)$ & \\
$(95 \%)$ & & \\
\hline
\end{tabular}

Quantitative determination of tumor necrosis factor (TNF- $\alpha$ ) in serum samples using ELISA and Relation between levels of TNF- $\alpha$ and $T B$ antigen.

In tuberculosis, $\mathrm{T}$ cells are responsible for protection, but also the pathology caused by inflammatory responses. Most T cells are divided into Th1 and Th2 subsets depending on the types of cytokines produced. Tumor necrosis factor is required for appropriate chemokine expression by 
M. tuberculosis-infected macrophages, both in vitro and in vivo. The serum levels of TNF- $\alpha$ were $83.4 \pm 12.3$ in patients positive for -TB circulating antigen, with a statistical significant difference $(\mathrm{P}<$ 0.0001) from the levels in negative individuals for TB $(19 \pm 1.73)$ as shown in table 7.

Table 7. The statistical significance of TNF- $\alpha$ cytokine in serum samples of $\mathrm{TB}$ patients positive and negative for TB-antigen.

\begin{tabular}{|l|l|l|}
\hline \multicolumn{2}{|c|}{$\begin{array}{l}\text { Serum levels }(\text { Mean } \pm \text { SD, } \\
\mathrm{pg} / \mathrm{ml}) \text { of }\end{array}$} & \multirow{2}{*}{ TNF $\alpha$ cytokine } \\
\cline { 1 - 2 } $\begin{array}{l}\text { TB-Ag }-\mathrm{Ag} \\
\text { Negative }(\mathrm{n}=\end{array}$ & $\begin{array}{l}\text { TB-Ag } \\
\text { Positive }(\mathrm{n}=\end{array}$ & \\
$20)$ & $20)$ & \\
\hline $19.0 \pm 1.7$ & $83.4 \pm 12.3$ & $<0.0001$ \\
\hline
\end{tabular}

\section{Discussion}

The often diagnosis of TB depends on invasive procedures such as laparoscopic peritoneal biopsy and culture. However, the availability of peritoneal biopsy is often limited due to underlying comorbidity ${ }^{[6]}$. Therefore, non-invasive, rapid, sensitive and specific diagnostic tests for TB are urgently required. In some studies they assessed the clinical usefulness of the recently developed $\mathrm{T}$ cell-based ELISPOT assay in patients with suspected TBP. We found that the PBMC ELISPOT assay (cutoff _ 6 spots) in patients with suspected TBP had a sensitivity of $86 \%$ for diagnosing active $\mathrm{TB}$, which is consistent with our previous report showing that the sensitivity of the PBMC ELISPOT assay for diagnosis of abdominal TB was $89 \%{ }^{[7]}$. It has been proposed that the diagnostic sensitivity for active TB of interferongamma release assays of blood is reduced by homing of sensitized $\mathrm{T}$ cells to the site of infection, as well as impaired cellular immunity in some patients ${ }^{[8]}$.

Cell-mediated immune responses are essential for protection against tuberculosis (TB) ${ }^{[9]}$. CD8+ T-cells produce cytotoxic molecules as well as cytokines. Cytotoxic T-cells are capable of killing cells infected with Mycobacterium tuberculosis (MTB) ${ }^{[10]}$, while aiding the recruitment and activation of other immune cell types. CD8+ T-cells recognize short antigenderived peptides (epitopes) presented on major histocompatibility complex (MHC) class I molecules on the surface of antigenpresenting cells (APCs) ${ }^{[11]}$. Clinical and preclinical assessment of CD8+ T-cells in a TB show that CD8+ T-cells play an important role in recall responses, as well as in long-term protection to MTB infection $^{[12]}$.

Interferon- $\gamma$ release assays (IGRAs) are the first new diagnostic test for latent tuberculosis (LTBI) in >100 years. Newest generation IGRAs measure interferon (IFN)- $\gamma$ secretion after exposure of whole blood (QuantiFERON-TB Gold In-Tube [QFT-GIT], Cellestis) or peripheral blood mononuclear cells (T-SPOT.TB [T-SPOT], Oxford Immunotec) to antigens encoded in the region of difference-1 (RD1), a portion of the Mycobacterium tuberculosis genome absent among all bacille Calmette-Guérin (BCG) strains and most nontuberculous mycobacteria [13]. We have shown in previous systematic reviews that compared with the tuberculin skin test (TST), IGRAs have higher specificity for LTBI in settings with low tuberculosis incidence, better correlation with surrogate measures of $M$. tuberculosis exposure, and less crossreactivity with the BCG vaccine. Thus, in recent years, IGRAs have become widely endorsed in high-income countries for diagnosis of LTBI ${ }^{[14-15]}$

In recent researches T-SPOT.TB shows good performance in detection of active tuberculosis during pregnancy. Interferon gamma release assay for $\mathrm{TB}$ screening of pregnant women is recommended in clinical practice because it may be a more appropriate diagnostic tool than the tuberculin skin test.

Interferon-gamma release assays (IGRAs), such as QuantiFERON Gold Test In-Tube (QGIT; Cellestis) and T-SPOT.TB (Oxford Immunotech), measure IFN- $\gamma$ released from peripheral blood mononuclear cell (PBMC) exposed to TBspecific antigens. For detection of latent TB in pregnancy, IGRA showed good performance, while concordance and the agreement were poor when comparing the tuberculin skin test (TST) and IGRA results [16]. To avoid false-positive interpretations, TST is discouraged and IGRA is recommended for $\mathrm{TB}$ screening and diagnosis in pregnant populations with high BCG vaccination coverage or uncertain vaccination status. 
Previously, many guidelines for the diagnosis of LTBI have relied on the tuberculin skin test (TST), despite its limitations. The TST may produce falsepositive results owing to prior Bacillus Calmette-Guérin (BCG) vaccination or nontuberculous mycobacterial infection; this poor specificity can lead to unnecessary LTBI treatment, with the risk of drug toxicity [17]. Moreover, either the inflammatory disorder itself or the immunosuppressive treatment may lead to false-negative TST results ${ }^{[18] .}$

In a another study makes Screening in Arthritis Patients for Latent Tuberculosis Infection before Starting Antitumor Necrosis Factor Treatment study, approximately $30 \%$ of patients with RA or AS were diagnosed with LTBI using the IGRA and received LTBI treatment. During a 41.7-month median follow-up period, five patients $(1.5 \%)$ developed active TB in a median of 20.8 months after initiation of anti-TNF treatment. The incidence rate of TB during the follow-up period did not differ between TST+/QFTpatients and TST-/QFT - patients, although all patients did not receive LTBI treatment. This study suggested that IGRA could be used instead of TST for diagnosing LTBI in patients before starting anti-TNF treatment in countries such as Korea, where the TB prevalence is intermediate and the BCG vaccination is mandatory at birth ${ }^{[19]}$. Investigation of LTBI in arthritis patients who received anti-TNF treatment is very important because such patients are at increased risk of LTBI reactivation ${ }^{[20] . ~ T o ~}$ prevent reactivation of LTBI, a number of countries have generated national guidelines to address LTBI before initiation of anti-TNF therapy. However, LTBI screening with TST has several drawbacks, such as a potential for false-positive results in individuals who have receive a BCG vaccination, particularly given after infancy and a potential for false-negative results in individuals with impaired cellular immunity ${ }^{[21]}$. Considering that the TST has a low specificity for LTBI diagnosis and its result is affected by many other factors, IGRA is regarded as an alternative to TST in the diagnosis of LTBI, especially in patients who have previously received a BCG vaccination [22].
The vast majority of the estimated annual 9.4 million new cases of active tuberculosis and 1.7 million tuberculosisrelated deaths occur in low- and middleincome countries [23]. Because of resource constraints, public health policies have appropriately placed limited emphasis on diagnosis and treatment of LTBI in these settings. Clinical use of IGRAs, however, has expanded dramatically in recent years, especially in the private sector ${ }^{\text {[24]. Because }}$ of their high burden of disease and emerging economies, these countries (eg, India, South Africa, Brazil, and China) represent a potentially lucrative market for commercial IGRAs. Although IGRAs are intended for LTBI and not an active tuberculosis disease, and although these tests cannot distinguish between latent infection and active disease, there is concern about the increasing use of IGRAs for active tuberculosis in high-burden countries. In this systematic review focused on individuals living in low- and middleincome countries, the highest-quality evidence from persons with suspected tuberculosis demonstrated sensitivity of $69 \%-83 \%$ and specificity of $52 \%-61 \%$ for IGRAs in the diagnosis of active tuberculosis. Furthermore, there was no consistent evidence that either IGRA was more sensitive than the TST for active tuberculosis diagnosis.

The majority of evidence for the diagnostic accuracy of IGRAs to date have been summarized from highincome settings where active tuberculosis has been used as a surrogate reference standard for LTBI diagnosis ${ }^{[25] .}$ However, diagnostic test performance (eg., sensitivity and specificity) can be expected to vary according to disease prevalence and other population characteristics [26]. Likewise, clinicians have been advised to base their decisions on studies that most closely match their own clinical circumstances ${ }^{[27] .}$

IGRAs were designed as diagnostic tests of LTBI, though the lack of an accepted gold standard for LTBI has been a significant limitation in establishing test performance. In contrast, adequate and 
commonly used reference standards exist for diagnosing active tuberculosis. Among studies that enrolled persons with suspected active tuberculosis (i.e., patients with diagnostic uncertainty), both IGRAs demonstrated suboptimal rule-out value for active tuberculosis. In other words, approximately 1 in 4 patients with cultureconfirmed active tuberculosis can be expected to have negative IGRA results in low- and middle-income countries; this has consequences for patients in terms of morbidity and mortality. Although highquality data were limited, sensitivity of both IGRAs was lower among HIVinfected patients $(60 \%-70 \%)$, suggesting that $\sim 1$ in $3 \mathrm{HIV}$-infected patients with active tuberculosis will have negative IGRA results. The few available comparisons between QFT-GIT and TSPOT revealed higher sensitivity for the TSPOT platform, although this difference did not reach statistical significance. Lastly, comparisons with pooled estimates of TST sensitivity were difficult to interpret because of substantial heterogeneity. Our results, however, suggest that neither IGRA platform may be more sensitive than the TST for active tuberculosis diagnosis in low- and middle-income countries [28]. IGRA specificity in diagnosing LTBI, estimated among individuals at low risk for tuberculosis exposure in settings with low tuberculosis incidence (high-income settings), is known to be high ( $\geq 98 \%)^{[29]}$. In contrast, specificity for active tuberculosis diagnosis is best estimated only in studies evaluating persons with suspected tuberculosis. As expected, because of the high background LTBI prevalence and the known inability of IGRAs to differentiate LTBI from active tuberculosis [30], the specificity of both IGRAs for active tuberculosis was low, regardless of HIV status. These data suggest that 1 in 2 patients without active tuberculosis will have positive IGRA results; this has consequences for patients because of unnecessary therapy for tuberculosis and its attendant risks. Studies demonstrating activated T-cell IFN- $\gamma$ response throughout the entire spectrum of tuberculosis, from latent to active disease [31], lend biologic plausibility to our findings. Even in the spectrum of latent tuberculosis infection ${ }^{[32]}$, activated T-cell IFN- $\gamma$ responses occur throughout each phase, with the possible exception of the innate immune response (which eliminates $M$. tuberculosis without priming a T-cell immune response).

Comparisons of TST and IGRA Sensitivity: Many studies reported comparisons of TST and IGRA (3 T-SPOT and 6 QFT-GIT) sensitivity. TST sensitivity in the 5 studies ${ }^{[33,34]}$ involving HIV-uninfected patients was higher (78\%; 95\% CI, 71\%-86\%) than that in the 4 studies ${ }^{[35]}$ involving HIV-infected patients (45\%; 95\% CI, 15\%-75\%). IGRA sensitivity was not statistically different from TST sensitivity for either T-SPOT (sensitivity difference, $23 \%$; 95\% CI, 0\%$45 \% ; P=.05$ ) or QFT-GIT (sensitivity difference, $7 \%$; $95 \% \mathrm{CI},-9 \%$ to $23 \% ; P=$ .37) (Figure 4). There was significant heterogeneity for both estimates (I-squared, $>75 \% ; P<.001)$. Data were insufficient to form HIV-stratified pooled sensitivity differing estimates for either IGRA. Sensitivity difference between interferon- $\gamma$ release assay (IGRA) and tuberculin skin test (TST) results. The forest plots display percent different (IGRA sensitivity-TST sensitivity) for confirming active pulmonary tuberculosis in the individual. All specificity estimates were determined in persons with suspected tuberculosis with use of HSROC and/or bivariate techniques. Overall, pooled specificity was low for both T-SPOT $(61 \% ; 95 \%$ CI, 40\%-79\%; 6 studies) and QFT-GIT (52\%; 95\% CI, $41 \%-62 \%$; 8 studies). When restricted to HIV-infected persons with suspected active tuberculosis, pooled specificity for $\mathrm{T}$ SPOT (52\%; 95\% CI, 40\%-63\%; 4 studies $[36,37$,$] was similar to that for QFT-GIT$ (50\%; 95\% CI, 35\%-65\%; 5 studies ${ }^{[38,39]}$. An insufficient number of studies were available to estimate pooled specificity for HIV-uninfected patients.

\section{Conclusion}

The identified antigen in serum samples of pulmonary tuberculosis using TB-Spot ELISA technique. The sensitivity, specificity, efficiency of Ziehl-Neelsen stain were $48 \%, 100 \%$ and $64 \%$, while the sensitivity, specificity and efficiency of TB-Spot ELISA for the detection of antigen were $97 \%, 95 \%$ and $98 \%$. The serum 
levels of TNF- $\alpha$ reach statistical significant difference $(\mathrm{P}<0.0001)$ in patients positive for $\mathrm{TB}$ antigen in comparison with the levels in negative individuals for TB. Hence we consider SPOT-ELISA method for tuberculosis antigen detection in pulmonary tuberculosis as a routine test for the early and rapid diagnosis of tuberculosis. Serum concentrations of tumor necrosis factor may serve as markers of disease activity of TB. It can be easily performed in any routine clinical laboratory, and is best suited to tie laboratory in the developing countries where laboratory resources are limited. TSPOT.TB shows good performance in detection of active tuberculosis. Interferon gamma release assay for TB screening is recommended in clinical practice because it may be a more appropriate diagnostic tool than the tuberculin skin test.

\section{Acknowledgments}

This work was supported by a grant from Damietta University, Faculty of Science, Botany and Microbiology Department.

\section{References}

1. Babita Sharma, Nita Pal, Bharti Malhotra, and Leela Vyas. Evaluation of a Rapid Differentiation Test for Mycobacterium tuberculosis from other Mycobacteria by Selective Inhibition with p-nitrobenzoic Acid using MGIT 960.J. Lab. Physicians. 2012; 2 (2): 8992

2. Siu GK, Zhang Y, Lau TC, Lau RW, Ho PL. Mutations outside the rifampicin resistance-determining region associated with rifampicin resistance in Mycobacterium tuberculosis. J Antimicrob Chemother. 2011; 66: 730-733.

3. . Lalvani A, Pareek M.. Interferon gamma release assays: principles and practice. Enferm Infec Microbiol Clin. 2010; 28(4):245-52.

4. Jafari C, Thijsen S, Sotgiu G, Goletti D, Domı'nguez Benı'tez JA,Losi M, Eberhardt R, Kirsten D, Kalsdorf B, Bossink A,. Bronchoalveolar lavage enzyme-linked immunospot for a rapid diagnosis of tuberculosis: a Tuberculosis Network European
Trials group study. Am J RespirCrit Care Med; 2009; 180: 666-673.

5. Mazurek GH, Jereb J, Vernon A, LoBue P, Goldberg S. Updated guidelines for using Interferon Gamma Release Assays to detect Mycobacterium tuberculosis infection - United States. MMWRRecomm Rep. 2010; 59: 1-25

6. Riquelme A, Calvo M, Salech F, Valderrama S, Pattillo A, Arellano M. Value of adenosine deaminase (ADA) in ascetic fluid for the diagnosis of tuberculosis peritonitis: a metaanalysis. J Clin Gastroenterol 2006;40: 705,10 .

7. Kim SH, Cho OH, Park SJ, Ye BD, Sung H, Kim MN. Diagnosis of abdominal tuberculosis by T-cellbased assays on peripheral blood and peritoneal fluid mononuclear cells. J Infect 2009;59: 409,15.

8. Joshi R, Pai M. Can pleural tuberculosis be diagnosed using interferon-gamma release assays? Respiration 2008;76: 128,30.

9. O'Garra A, Redford PS, McNab FW, Bloom CI, Wilkinson RJ, Berry MP. The immune response in tuberculosis. Annu Rev Immunol 2013;31: 475-527

10. Prezzemolo T, Guggino G, La Manna MP, Di Liberto D, Dieli F, Caccamo $\mathrm{N}$. Functional signatures of human CD4 and CD8 $\mathrm{T}$ cell responses to Mycobacterium tuberculosis. Front Immunol 2014;5: 180.

11. Abbas A, Lichtman A, Pilai S. Basic immunology: functions and disorders of the immune system. Philadelphia: Elsevier Saunders; 2014.

12. Behar SM. Antigen-specific CD8(+) T cells and protective immunity to $\mathrm{M}$. tuberculosis. Adv Exp Med Biol 2013;783: 141-63.

13. Pai M, Kalantri S, Dheda K. New tools and emerging technologies for the diagnosis of tuberculosis: part I. Latent tuberculosis. Expert Rev Mol Diagn. 2006;6: 413-22.

14. Mazurek G, Jereb J, Vernon A, LoBue $\mathrm{P}$, Goldberg S. Updated guidelines for using interferon-gamma release assays to detect Mycobacterium tuberculosis infection, United States. MMWR.

15. NHS. Health Protection Agency position statement on the use of interferon gamma release assay (IGRA) tests for tuberculosis (TB) 
HPA Tuberculosis Programme Board; 2008.

16. Lighter-Fisher J, Surette AM. Performance of an interferon-gamma release assay to diagnose latent tuberculosis infection during pregnancy. Obstet Gynecol. 2012; 119:1088-95.

17. Kim JH, Cho SK, Han M, Choi CB, Kim TH, Jun JB. Factors influencing discrepancies between the QuantiFERON-TB gold in tube test and the tuberculin skin test in Korean patients with rheumatic diseases. Semin Arthritis Rheum. 2013;42: 424432.

18. Ponce de Leon D, Acevedo-Vasquez E, Sanchez-Torres A, Cucho M, Alfaro J, Perich R. Attenuated response to purified protein derivative in patients with rheumatoid arthritis: study in a population with a high prevalence of tuberculosis. Ann Rheum Dis. 2005; 64: 1360-1361.

19. Hyun Lee, Hye Yun Park, Kyeongman Jeon, Byeong-Ho Jeong, Ji-Won Hwang, Jaejoon Lee, Hoon-Suk Cha,Eun-Mi Koh, Eun-Suk Kang, and Won-Jung Koh,. QuantiFERON-TB Gold In-Tube Assay for Screening Arthritis Patients for Latent Tuberculosis Infection before Starting Anti-Tumor Necrosis Factor Treatment Published online 2015.

20. Solovic I, Sester M, Gomez-Reino JJ, Rieder HL, Ehlers S, Milburn HJ. The risk of tuberculosis related to tumor necrosis factor antagonist therapies: a TBNET consensus statement. Eur Respir J. 2010;36: 1185-1206.

21. Mariette X, Baron G, Tubach F, Liote F, Combe B, Miceli-Richard C. Influence of replacing tuberculin skin test with ex vivo interferon $\gamma$ release assays on decision to administer prophylactic antituberculosis antibiotics before anti-TNF therapy. Ann Rheum Dis. 2012;71: 1783-1790.

22. Hsia EC, Schluger N, Cush JJ, Chaisson RE, Matteson EL, Xu S. Interferon- $\gamma$ release assay versus tuberculin skin test prior to treatment with golimumab, a human anti-tumor necrosis factor antibody, in patients with rheumatoid arthritis, psoriatic arthritis, or ankylosing spondylitis. Arthritis Rheum. 2012; 64: 20682077.
23. WHO. Global tuberculosis control 2010. Geneva: World Health Organization.

24. Denkinger CM, Dheda K, Pai M. Guidelines on interferon- $\gamma$ release assays for tuberculosis infection: concordance, discordance or confusion? Clin Microbiol Infect. 2011; 6: 806.

25. Diel R, Loddenkemper R, Nienhaus A. Evidence-based comparison of commercial interferon-gamma release assays for detecting active TB: a meta analysis. Chest. 2010; 137: 952-68.

26. Leeflang MM, Bossuyt PM, Irwig L. Diagnostic test accuracy may vary with prevalence: implications for evidence-based diagnosis. J Clin Epidemol. 2009; 62: 5-12.

27. Guyatt GH, Oxman AD, Vist GE. GRADE: an emerging consensus on rating quality of evidence and strength of recommendations. BMJ. 2008; 336: 924-6.

28. John Z. Metcalfe, Charles K. Everett, Karen R. Steingart, Adithya Cattamanchi, Laurence Huang, Philip C. Hopewell, and Madhukar pai Interferon- $\gamma$ Release Assays for Active Pulmonary Tuberculosis Diagnosis in Adults in Low- and Middle-Income Countries: Systematic Review and Meta-analysis. J Infect Dis. 2011 Nov 15; 204 (Suppl 4): S1120-S1129.

29. Pai M, Zwerling A, Menzies D. Systematic review: T cell-based assays for the diagnosis of latent tuberculosis infection: an update. Ann Intern Med. 2008;149:177-84.

30. Pai M, Menzies D. Interferon-gamma release assays: what is their role in the diagnosis of active tuberculosis? Clin Infect Dis. 2007; 44: 74-7.

31. Andersen P, Doherty TM, Pai M, Weldingh K. The prognosis of latent tuberculosis: can disease be predicted? Trends Mol Med. 2007; 13: 175-82.

32. Barry CE, 3rd, Boshoff HI, Dartois V. The spectrum of latent tuberculosis: rethinking the biology and intervention strategies. Nat Rev Microbiol. 2009; 7: 845-55.

33. Katiyar SK, Sampath A, Bihari S, Mamtani M, Kulkarni H. Use of the QuantiFERON-TB Gold In-Tube test to monitor treatment efficacy in active pulmonary tuberculosis. Int $\mathrm{J}$ Tuberc Lung Dis. 2008; 12: 1146-52. 
34. Ozekinci T, Ozbek E, Celik Y. Comparison of tuberculin skin test and a specific T cell-based test, T-Spot.TB, for the diagnosis of latent tuberculosis infection. J Int Med Res. 2007; 35: 696-703.

35. Jiang W, Shao L, Zhang Y. Highsensitive and rapid detection of Mycobacterium tuberculosis infection by IFN-gamma release assay among HIV-infected individuals in BCGvaccinated area. BMC Immunol. 2009; 10: 31.

36. Cattamanchi A, Smith R, Steingart $\mathrm{KR}$. Interferon-gamma release assays for the diagnosis of latent tuberculosis infection in HIV-infected individuals: a systematic review and meta-analysis. J Acquir Immune Defic Syndr. 2011; 56: $230-8$.
37. Leidl L, Mayanja-Kizza H, Sotgiu G. Relationship of immunodiagnostic assays for tuberculosis and numbers of circulating CD4+ $\mathrm{T}$ cells in HIVinfection. Eur Respir J. 2010; 35: 61926.

38. Kabeer BSA, Sikhamani R, Swaminathan S, Perumal V, Paramasivam P, Raja A. Role of interferon gamma release assay in active TB diagnosis among HIV infected individuals. PLoS One. 2009; 4: 5718.

39. Leidl L, Mayanja-Kizza H, Sotgiu G. Relationship of immunodiagnostic assays for tuberculosis and numbers of circulating CD4+ $\mathrm{T}$ cells in HIVinfection. Eur Respir J. 2010; 35: 61926.

\title{
الملخص العربي
}

عنوان البحث: دراسة علي اختبار وتعريف بعض أنواع ميكوباكتيريم بإستخدام بعض التقتيات البكتيريولوجية والمناعية

\author{
محمد إسماعيل ابو دباره و اميره علي الفلال و نوران صابر المليجي \\ قسم النبات والميكروبيولوجى- كلية العلوم -جامعة دمياط
}

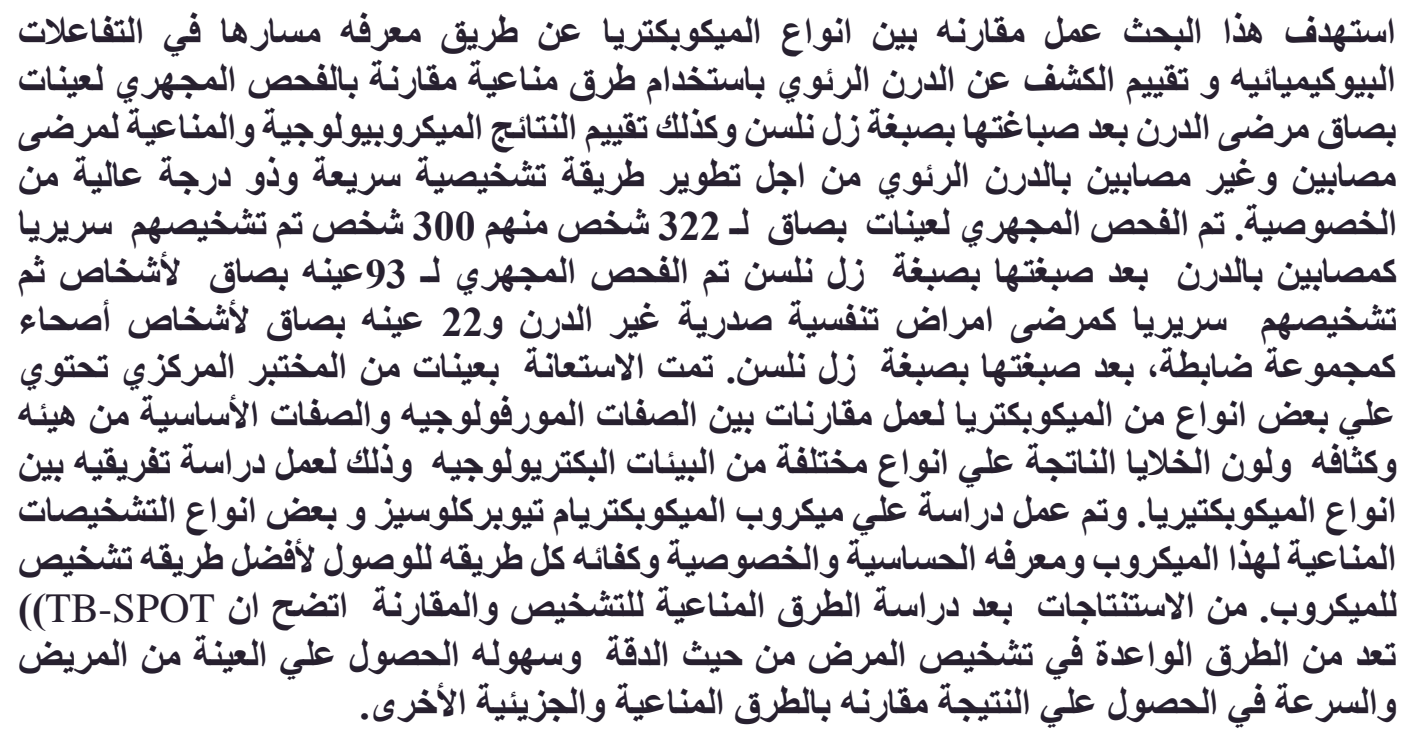

